\title{
Micro-miniaturized electrophoresis DNA Separator using MEMS
}

\author{
Prakash R. Apte*, Litan Kumar Mohanta ${ }^{\dagger}$, Amit C. Vartak*, \\ $*$ Department of Electrical Engineering, IIT Bombay, India. $\dagger$ Department of Mechanical \\ Engineering, IIT Bombay, India.
}

\begin{abstract}
Conventional DNA separation procedures involve large sizes, high voltages and are unfit for large molecules. Proposed set-up constitutes two fold separation techniques, porous filter for fractionating strands on size and final run through microchannels (agarose, buffer solution) viscous enough for DNA electrophoresis. For arriving at the final set-up all the physical contradictions like voltage, viscosity of the fluid, length of the channel were analyzed.

The physical set up consists around 20 microchannels (varying diameters) positioned at 500um (centre-to-centre spacing) assembling entire device within $1 \mathrm{~cm}$. Polymerase Chain Reaction (PCR) treated DNA assays, are fed to microchannel entrances. Mixture of DNA strands are then passed through magnetic filters. Filtering property of the filters is adjusted by regulating corresponding magnetic field strengths. Smallest strands pass through small pored filter, owing to high velocity (in electrophoresis), thus categorization being done. Proposal replaces conventional apparatus by miniaturized equipment, in ideal case disposable. Miniaturization reduced voltages requirement, solving high-voltage handling problems. Proposed apparatus can fractionate large (>200kbp) molecules and even organic molecules.
\end{abstract}

Key Words: microchannel, multi-channel, electrophoresis, miniaturization, DNA separation

\section{INTRODUCTION}

DNA array technologies provide rapid and cost-effective methods of identifying genetic variations and gene expressions. DNA separation has a growing importance in numerous applications in biotechnology, medicine and chemistry. Wealth of available DNA sequence data makes it possible to identify many diseases as well as biological threats such as the presence of infectious agents in the environment. Alterations in gene expression patterns or in a DNA sequence can seriously affect biological functions. Miniaturizing the process could decrease the cost and timescale of gene sequencing. Microfabricated bioanalytical devices are very efficient platforms for simultaneous analysis of biologically important molecules; and they offer great potential for sensitive and large-scale DNA analysis.

Efforts to improve DNA separation in microdevices have led to advances in capillary electrophoresis and the development of novel separation strategies. Microfluidics focused on the development of separation matrices with low injection viscosities has been recently developing. Small sample volumes and increased separation speed have made microscale separation strategies the focus of current research. The sequencing of genomes requires analysis of a wider range of DNA fragments, therefore, research will focus on methods for designing tunable sieving matrices or devices with tunable pore size ${ }^{1}$.

Microfabricated bioanalytical devices, frequently referred to as "lab-on-a-chip", typically comprise microchannel networks, miniaturized polymerase chain reaction chambers and/or restriction digestion reactors, microseparation units and possible combinations of the above to accommodate DNA analysis ${ }^{2}$. Some of the main advantages of miniaturization include improved performance, separation speed and throughput, reduced costs and reagent consumption, as well as integrated and parallel analysis for high-throughput environments. Authors propose an appropriate miniaturization of the strand separation process.

The main aim of the conventional apparatus is to separate the DNA strands according to their sizes in the capillary tube so that the DNA in the test sample can be compared with the reference sample for similarities. Generally the method used for the separation is electrophoresis. Electrophoresis is accomplished by inducing the migration of charged molecules in an electrolyte under the influence of electric field. Smaller or highly charged molecules move faster than the larger or lower charged molecules. By utilizing this technique each species of the DNA sample molecule in divided 
in to bands that pass a particular point at different time. Once these fragments pass the fixed point they are analyzed by the detection method in order to sequence them.

\section{DIFFERENT METHODS USED FOR DNA SEPARATION}

Different types of electrophoresis are used in DNA sequencing. For example, in capillary electrophoresis, a buffer-filled capillary is suspended between two reservoirs of buffer. An electric field is applied to both ends of the capillary to create a bias across the length of the capillary. A sample is introduced at one end of the capillary. The sample migrates according to the physical characteristics of the DNA fragments and separate along the electric field bias created.

As a further example, the standard high speed automated DNA sequencers make use of a thin gel layer, of several hundred microns, encased between two etched glass plates in order to separate the fluorescent dye labeled DNA fragments by an applied electric field ${ }^{3}$. The separation of the DNA fragments depends on their relative lengths, which can vary by as little as one chemical base. Making use of enzyme chemistry and labeling each of the four bases, by terminating a given DNA fragment with a differently colored dye, the process is accomplished.

As is understood, using any of these electrophoresis methods, each fluorescent dye label can be individually detected by a detector. Once a DNA fragment migrates, it is detected by the detector and then the identity of the base is determined based on the fluorescent dye label. For detection the most commonly used technology is a four-color method that uses four standard dyes for each of the chemical bases of DNA. Other detection methods include the fluorescent target detection.

Among recent developments are-"microfabricated Hexagonal lattices using spatially asymmetric pulsed field electrophoresis" ${ }^{4}$.using micro-fabricated current injectors, which can fractionate samples in a few seconds but they are valid only for 50-200 kbp.

Developments have also been done using molecular sieving techniques ${ }^{5}$, involving separation method and a genetic analysis method using microfluidics. This method enables separation of complexes that are formed at the "differential medium laminar interface" of a microchannel by simple use of syringe pumping. That is, this separation is accomplished by molecular sieving effect microfluidics. Analysis of the motion of a single DNA molecules have been previously carried out on device made of polydimethylsiloxane(PDMS) ${ }^{6}$ that has a micro-channel in which arranged in a hexagonal lattice. Electrophoresis of DNA in the pillars showed the average speed of DNA migration was dependent on its lengths. To measure the speed of DNA motion in each showed the dependence of length on the mobility of space and gap.

\section{DESIGN ISSUES IN THE ELECTROPHORESIS APPARATUS}

For DNA separation by electrophoresis for optimal design many conflicting design issues have to be handled. The main parameters that are to be optimized are diameter of the capillary tube, viscosity of the gel used, voltage that is applied and how long the channel is required for better separation. For designing the optimized instrument each of the parameters is to be well analyzed for the ideal level required (fig. 1) and then the cross-conflicts are to be resolved.

The above mentioned conflicting parameters generally have two possible levels e.g. in case of length of the channel, it has to be large enough for better separation, at the same time, for miniaturization, the channel has to be as small as possible. Here both the things i.e. miniaturization and better separation are required. One design approach can be choosing optimum level of the channel so that a compromise is made between both of the parameters. Other approach can be holding the length at one level and changing some other parameter to adjust the contradictory requirement. Fig. 1 shows length and voltage as two physical contradictions. Lower voltage assists ease in handling and power consumption, while the higher voltage improves the speed of separation. Now if one chooses smaller length channel with low voltage, then, miniaturization and ease in handling are achieved. Smaller channel implies lesser time for traveling, so the advantage of larger voltage is also embedded in the choice. 


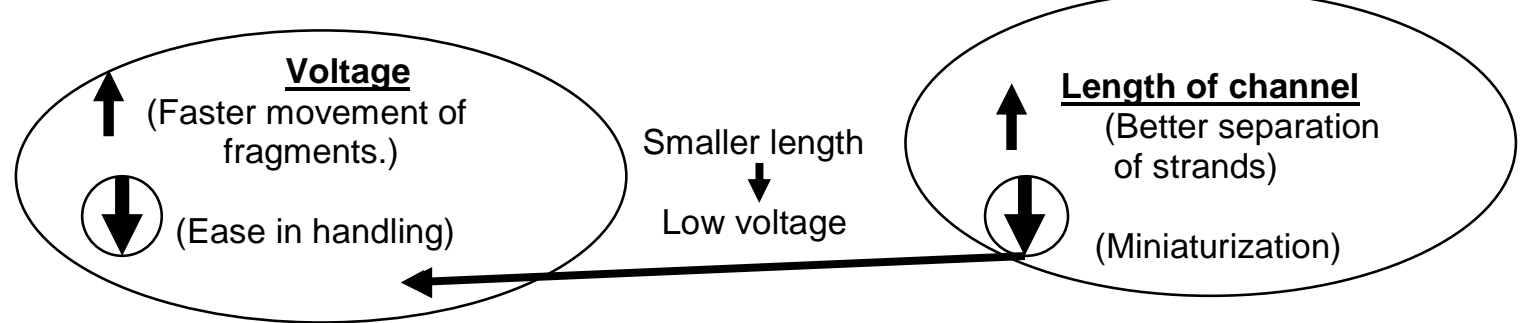

By choosing smaller channel and lower voltage the advantage of higher voltage is also achieved.

Fig.1 Length and voltage as two physical contradictions with the conflicts.

The bullets shows all four conflicts and there required values.

- The length of the channel is to be kept small for the miniaturization of the device but for better separation of the DNA strands the longer channels are favorable.

- Voltage directly affects the movement of the fragments. With higher voltages the DNA samples can reach other end faster. But lower voltages are better for the handing of the device and as well the joules heating is less with lower voltages.

- For producing larger diameter tubes the standard manufacturing processes can be used but smaller diameter reduces the requirements of the DNA samples and also the current and power losses are minimized.

- Viscosity of the gel decides the size of the fragments reaching at the end of the tube in given amount of time. Highly viscous fluids assist the separation of smaller fragments i.e. smaller fragments will reach the other end but larger will hardly start the journey. Conversely in lower viscous medium, by the time larger fragments have reached the end smaller have already escaped out.

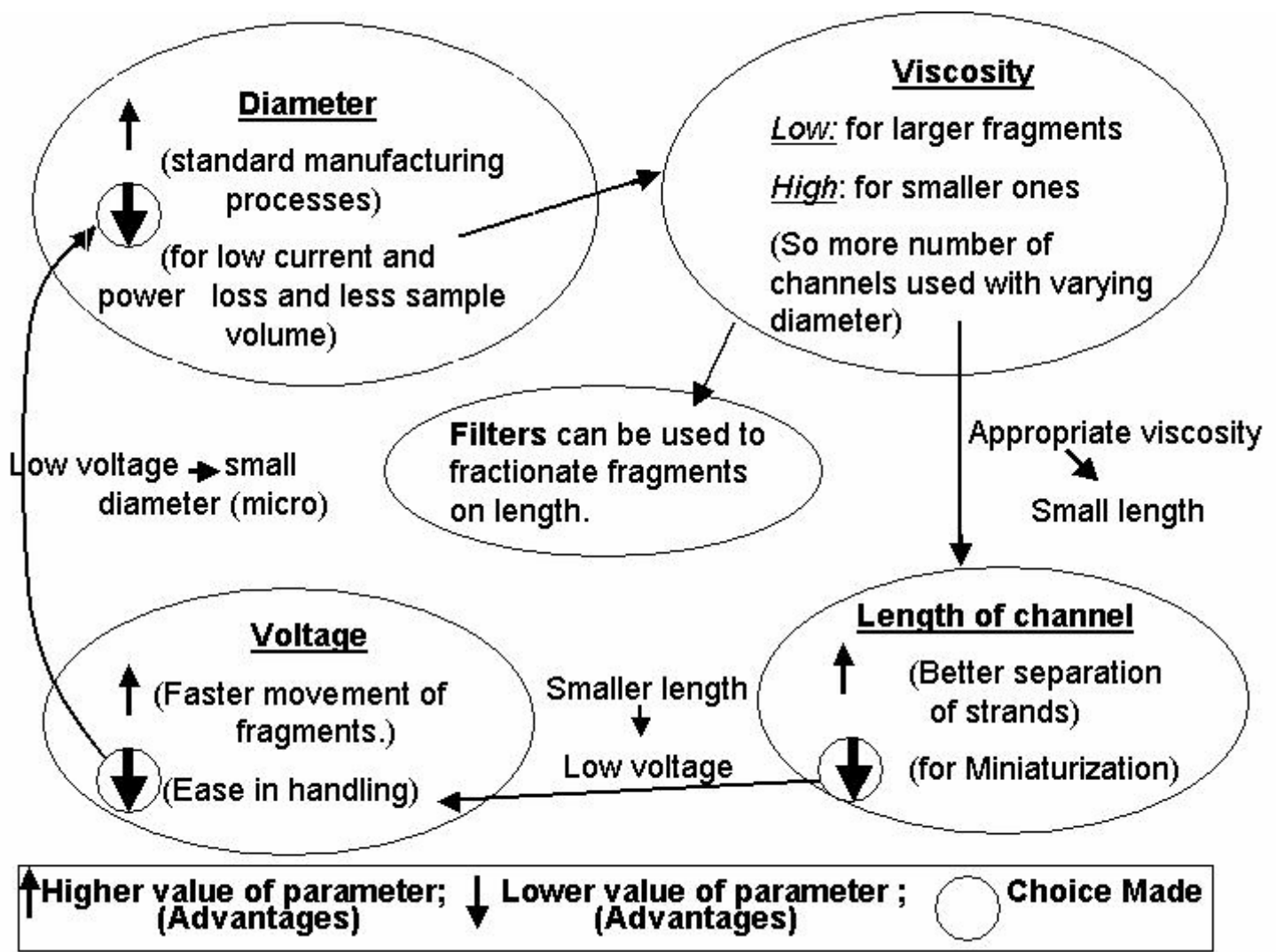

Fig. 2 Optimization of the conflicting parameters. 
In summary we want a device with smaller dimension, lower voltage requirements and different viscosities to separate different DNA strands. In the apparatus authors have selected the above parameters in such a way so that the main function of separation of DNA is carried out in efficient manner. The supply potential has to be reduced for better handling and low joule heating of the apparatus. But with lower voltage more time is required for passing the DNA. This problem can be solved by keeping the channel length small, so that the time required to travel would be less. By reducing the channel length the DNA separation also decreases. Changing viscosity can enhance the separation. It would be ideal to have varying viscosities, so that in the channel with lower viscosity larger fragments can be separated and in higher viscous channel smaller fragments are divided. By changing the diameter of the tubes the flow of DNA can be changed to get good separation

\section{THE PROPOSED SETUP}

The setup constitutes of two fold separation technique, porous filter for fractionating strands on size and final run through microchannels (agarose, buffer solution) viscous enough for DNA electrophoresis (Fig.3). The array of porous filters is used for the filtration. The first filter has the finest grid size and the subsequently filters grid size increases. Due to the finer grid, the first filter will fractionate only the smallest size DNA fragments and all the higher size DNA fragments will be passed away to the next coarser filter. Like wise every filter accepts the DNA sizes of its pass band and the higher sized samples are passed to the next filter. As a analogy suppose there are 100 fragments of different sizes, and the filter array consists of 20 filters, then in the ideal case the first filter will pass first five samples(1-5), next filter (6-10) and so on and so forth. Observe that any sample passed through a multi-channel consists of only immediate "size-neighbors", facilitating personalized separation of particular lot, i.e. low viscous channel for large fragments and contrariwise.

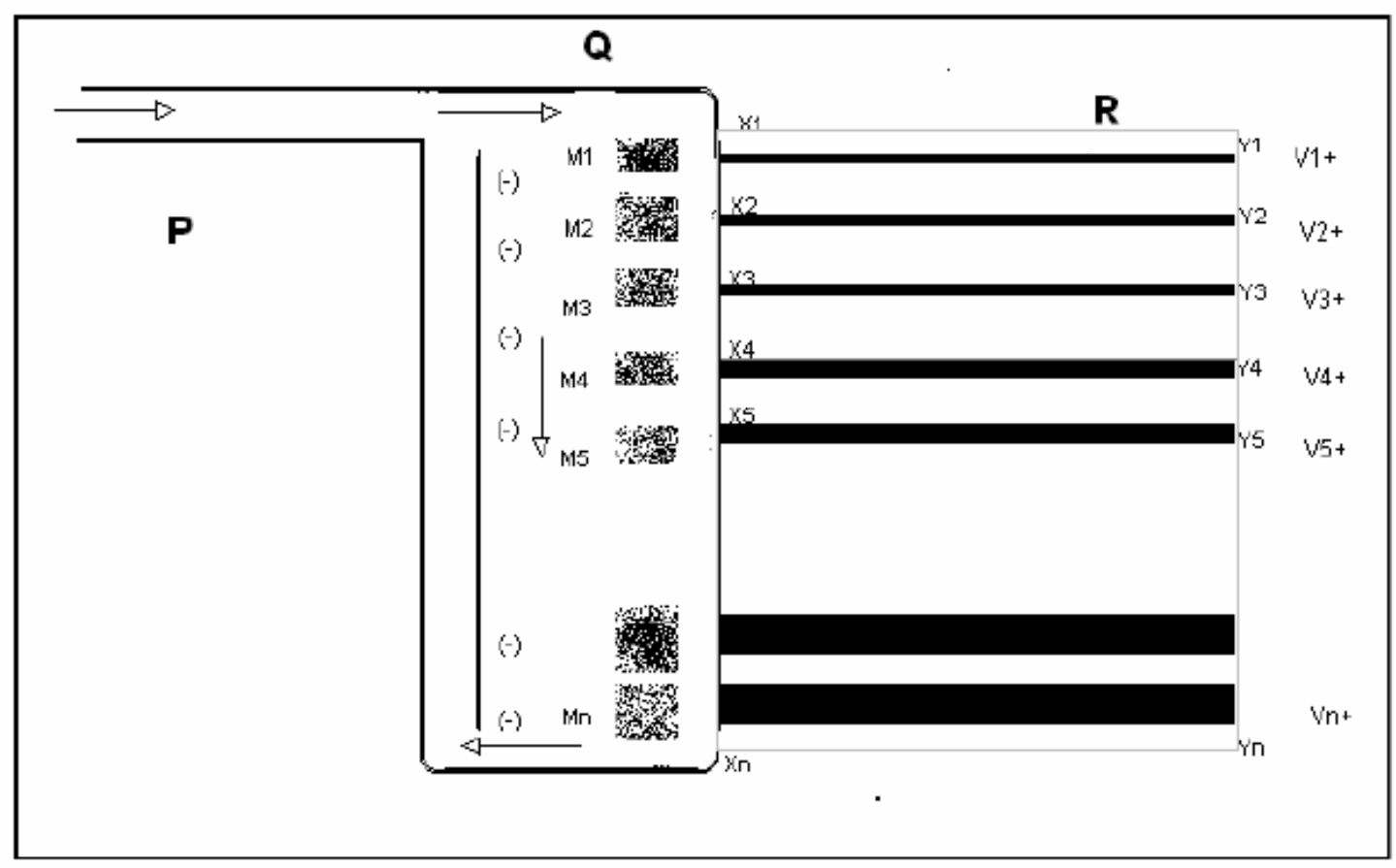

Fig. 3 Proposed setup for DNA separation

At the output of each filter a channel of varying diameter is present. The channel is filled with the required concentration of agarose gel. The diameter of the channel decides the viscosity of the gel. Smaller DNA strands are present at the input of the first channel, hence the viscosity of the first channel should be high enough so that only smaller fragments are passed through the channel, and larger fragment can travel very small distance. The DNA fragment is said to be out of detection tolerance limit (DTL) once it has passed the $80 \%$ of the channel length. 
As filter becomes more and more porous the diameter of channel attached to it also increases, in turn increasing the viscosity. So in larger diameter channels the smaller size DNA strands if present will pass the DTL while in larger DNA's are still present in the DTL. While in the smaller diameter channel the larger DNA will experience more resistance to travel and will not reach up to DTL.

Considering aforementioned factors, the physical set-up consists around 20 microchannels of varying diameters positioned at 500um (centre-to-centre spacing) assembling entire device within $1 \mathrm{~cm}$. (Fig. 3). PCR treated DNA assays, are fed at P, flown to microchannel entrances X1, X2, X3 etc. Mixture of DNA strands are then passed through magnetic filters M1, M2 etc. Magnetic sand filters have peculiar property of varying the porosity by changing the magnetic field applied to it. So filtering property of the filters (pore sizes $\mathrm{M} 1<\mathrm{M} 2<\mathrm{M} 3$ etc) is adjusted by regulating corresponding magnetic field strengths.

\section{PHYSICAL SIZES AND SIMULATION RESULTS}

The above mentioned setup is simulated with the help of CoventorWare ${ }^{\mathrm{TM}}$ Version 2004 tool and analysis was done with the help of microfludics netflow solver. Firstly two channels one with length 200um and other with length 1000 um were simulated with 20um and 40um as their widths respectively. A bunch of DNA strands (with certain mobility) with Gaussian profile (sigma 20) was kept initially at one end (inlet) of the channel. Voltage of 3-5V was applied at the outlet, and simulation was initialized. After simulation run of 4 secs the position of the DNA bunch was located. The same simulation was performed for different DNA mobility values and each time end position of the bunch was noted.

For the channel length of $200 \mathrm{um}$ with mobility variation from $5000 \mathrm{um}^{2} / \mathrm{V}$-s to $15000 \mathrm{um} 2 / \mathrm{V}$-s, the separation was not enough to detect every bunch separately. For higher end motility values, DNA fragments went out of the capillary, while for lower values exact separation was not clear due to diffusion coefficient of DNA. One thing can be concluded from these simulations, separation was linearly varying with the mobility.

To increase the separation the channel length was increased to $1000 \mathrm{um}$ and the same simulation with voltage of $5 \mathrm{~V}$ was performed. The distance traveled by the DNA with different mobility and for two different voltages is given in Table 1.

\begin{tabular}{|l|c|c|}
\hline \multirow{2}{*}{$\begin{array}{l}\text { Mobility } \\
\mathrm{um}^{2} \mathrm{~V}^{-1} \mathrm{~s}^{-1}\end{array}$} & \multicolumn{2}{|c|}{$\begin{array}{c}\text { Distance Traveled } \\
\text { (um) }\end{array}$} \\
\cline { 2 - 3 } & 3 Volt & 5 Volt \\
\hline 14000 & 50 & 309 \\
\hline 15000 & 54 & 332 \\
\hline 16000 & 57 & 345 \\
\hline
\end{tabular}

Table 1. Distance traveled by the DNA in 1000um channel for varying voltages and motilities.

Table 2 shows the distance traveled by DNA with mobility of $15000 \mathrm{um}^{2} / \mathrm{V}-\mathrm{s}$ in a 1000 um channel for varying voltages. Results were satisfactory with respect to separation but the distance DNA traversed was merely half of the length of capillary in 4-5 sec. (As shown in the table). One can utilize the full length of the tube either by increasing the voltage, or by increasing the time for simulation run, both giving un-necessary burdens. Decreasing the capillary dimensions to say half can give the desired separation with same time and voltage. 


\begin{tabular}{|c|c|}
\hline $\begin{array}{c}\text { Voltage Applied } \\
(\mathrm{V})\end{array}$ & $\begin{array}{c}\text { Distance Traveled } \\
\text { (um) }\end{array}$ \\
\hline 1 & 17 \\
\hline 3 & 57 \\
\hline 5 & 98 \\
\hline 7 & 141 \\
\hline 9 & 179 \\
\hline 10 & 209 \\
\hline 11 & 227 \\
\hline
\end{tabular}

Table 2 Relationship between voltage and distance traveled for 1000um channel.

Simulation results for effect of mobility on distance traveled for different voltages for the capillary length of 500um are shown in Fig. 4. A capillary tube with length 500um and etch depth of $15 \mathrm{um}$ was taken into consideration. A linear mesh with element size in cross section as $15 \mathrm{um}$ and in the flow direction as 5 um was generated as first approximation. The transient response for $4 \mathrm{sec}$. with output time of $0.5 \mathrm{sec}$ was observed. The DNA species introduced at inlet were assumed to have Gaussian distribution with located at $1 \mathrm{um}$ and sigma(x) as 20um. The distance traveled by the strand was plotted with respect to the mobility. This analysis was done for $3 \mathrm{~V}$ and $5 \mathrm{~V}$ voltage values.

The distance traversed by the strand is proportional to the mobility. From the graph it is highlighted that the slope of the curve with $5 \mathrm{~V}$ supply is more than $3 \mathrm{~V}$ supply, suggesting that separation is better for higher voltages. But the channel length and the voltage limitations must be taken in to consideration.

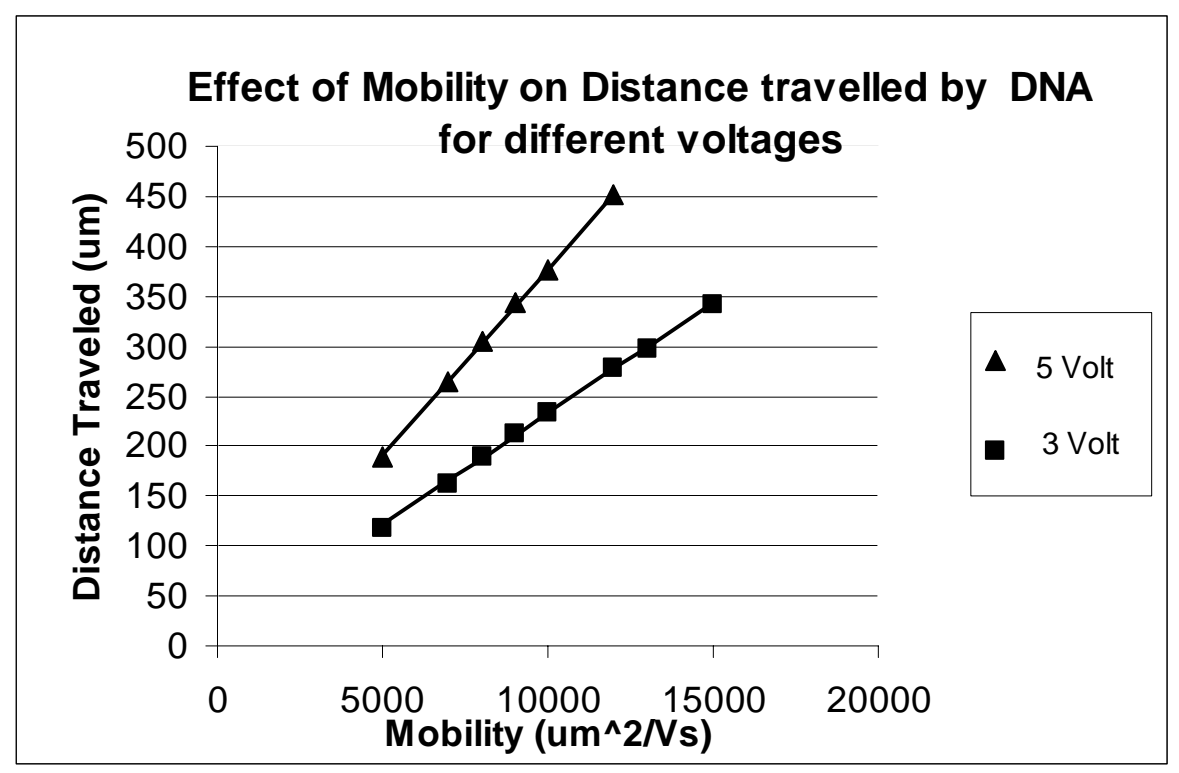

Fig. 4. Effect of Mobility on distance traveled by DNA for different voltages 
Fig.5 shows the pictorial view of DNA fragments in the capillary tube. The figure shows three different simulations for three mobility values. DNA is being inlet at the left end and after $4 \mathrm{~s}$ the position of the DNA is observed. The separation is clearly seen from the figure.

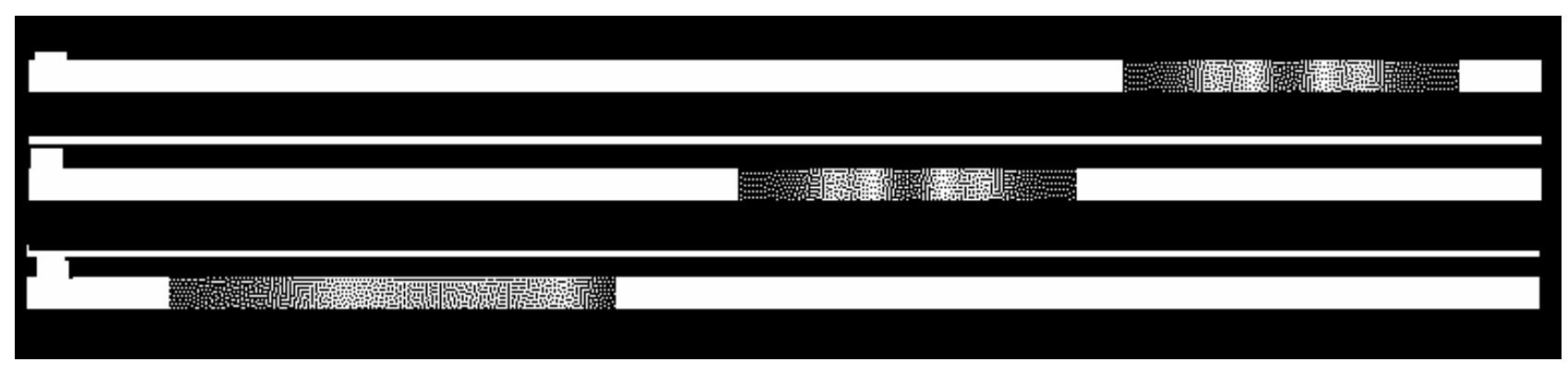

Fig. 5 Pictorial view of the tube with DNA samples separated after simulation

\section{DISCUSSION}

The important achievement of DNA separation with gel electrophoresis is the miniaturization. The size of the chip can be compressed to as low as 500um. The conventional electrophoresis requires 10's or 100's of volts for separation of DNA. In this method the authors have managed to reduce it up to few Volts by which the power requirements as well the heating of apparatus decreases.

It is observed from the simulation results that, for small length channels ( 200um) due to diffusion effects of DNA, the separation is not very good at low voltages. For better separation one can increase voltage but by this the fragments with lower mobility will completely escape the channel. Conversely if the length is increase too large( 1000um) the separation is better at the cost of more voltage or larger time. The optimum length of the channel is chosen as 500um where a considerable separation is achieved in 4-5 sec. Larger voltages assist separation. Magnetic filters substantially categorize the DNA and thus personalizing the work of separation. The applied voltage and the distance moved by the DNA strands are inversely proportional. Proposed apparatus can fractionate larger molecules (200kbp) and even organic molecules.

\section{REFERENCES}

[1] Randolph Ashton_, Chakradhar Padala and Ravi S Kane , "Microfluidic separation of DNA", Current Opinion in Biotechnology 2003, 14:497-504

[2] Shaorong Liu, Andras Guttman, “ Electrophoresis microchips for DNA analysis “ ,Trends in Analytical Chemistry, Vol. 23, No. 6, 2004

[3] Imager for DNA sequencer, United States patent no: 6800 438. Noolandi, et al. October 5, 2004.

[4] Austin R.,"A DNA Prism::rapid Continous Fractionation and separation of genomic DNA molecules",NNUN Abstracts 2002/Biology \& Chemistry, pp 6.

[5] Kenichi Yamashita, Yoshiko Yamaguchi, Masaya Miyazaki, Hiroyuki Nakamura, Hazime Shimizu, Hideaki Maeda, "Microfluidic system for DNA sequence detection" Chemical Engineering Journal (2004)

[6] Ken-ichi Inatomi, Shin-ichi Izuo, Sang-seok Lee, Hiroshi Ohji, Satoru Shiono. "Electrophoresis of DNA in micropillars fabricated in polydimethylsiloxane" Microelectronic Engineering 70 (2003) 13-18 